\title{
Efecto de marcar primero y la localización del partido en las principales ligas del fútbol europeo Effect of scoring first and match location in the main European football leagues *Francisco Daniel Martínez Martínez, **Higinio González García \\ *Consejería de Educación de la Región de Murcia (España), **Universidad Miguel Hernández de Elche (España)
}

Resumen. Los equipos de fútbol que se adelantan en el marcador tienen una mayor posibilidad de ganar el partido. Además, el fútbol es un deporte en el que se maneja un bajo número de goles. Por ello, los objetivos de este trabajo fueron: i) determinar la influencia del efecto de marcar primero, y la localización del encuentro en el resultado final de un partido de fútbol; y ii) contrastar la existencia de diferencias entre las principales ligas del fútbol europeo: LaLiga, Premier League, Francia Ligue 1, Bundesliga y Serie A. La muestra estuvo formada por todos los partidos $(N=1826)$ disputados durante la temporada 2016/2017 en la LaLiga, Premier League, Francia Ligue 1, Bundesliga y Serie A. La probabilidad de ganar el equipo local cuando marca primero fue significativamente mayor con respecto a la probabilidad de ganar cuando marca primero el equipo visitante $(t=12.69 ; p<.01 ; d$ $=.91$ ). En los resultados que se analizó la ventaja de marcar primero, cuando el equipo marca primero dentro y fuera de casa, se encontraron diferencias significativas ( $t=11.45 ; p<.01 ; d=.89$ ) a favor de los que juegan dentro de casa. Se concluyó que existe un gran equilibrio entre las principales ligas del fútbol europeo, así como la importancia que tiene para un equipo marcar el primer gol, ya sea en casa o a domicilio, pues le garantiza una alta probabilidad de obtener un buen resultado al final del partido.

Palabras clave: análisis del partido, ventaja, probabilidad, victoria, gol.

\begin{abstract}
Football teams taking the lead in the scoreboard have greater chance of winning the match. In addition, soccer is a sport in which there is a low number of goals. Thus, the objectives of this work were: i) to determine the influence of scoring first and the match location in the final result of football matches; and ii) to contrast the existence of differences between the main European football leagues: LaLiga, Premier League, French Ligue 1, Bundesliga, and Serie A. The sample consisted of all matches $(N=1.826)$ played during season $2016 / 2017$ in LaLiga, Premier League, French Ligue 1, Bundesliga, and Serie A. Results showed that the probability of the local team winning when they score first was significantly greater compared to the probability of winning when the visitant team score first $(t=12.69 ; p<.01 ; d=.91)$. There were found significant differences between home teams and visitant teams regarding scoring first teams regarding scoring first $(t=11.45 ; p<.01 ; d=.89$ ), in favour of local teams which showed higher possibilities to win. In conclusion, there is a great balance between the main European football leagues. Furthermore, scoring the first goal either at home or away guarantees a high probability of obtaining a good result at the end of the match.
\end{abstract}

Keywords: match analysis, advantage, probability, victory, goal.

\section{Introducción}

Marcar el primer gol en un partido es un importante predictor del resultado final en el fútbol profesional (Casal, Losada, Maneiro \& Ardá, 2017; Lago-Peñas, Gómez, Megías \& Pollard, 2016). La baja puntuación que se obtiene en el marcador de este deporte puede ser una de las razones que justifiquen la importancia de marcar primero (Castellano, 2009; López-García, Maneiro, Ardá, Rial, Losada \& Casal, 2018). De hecho, Anderson \& Sally (2014) indican que el número de goles marcados por partido en las principales ligas europeas es de 2.66. Como consecuencia, los equipos que anotan el primer gol ganan el 65-70\% de los encuentros (García-Rubio, Gómez, Lago-Peñas \& Ibáñez, 2015; Lago-Peñas et al., 2016; Sampedro \& Prieto, 2012).

Otro factor a tener en cuenta dentro del deporte de competición es la ventaja de jugar en casa, o Home Advantage (HA), que permite conocer qué efecto tiene competir en casa o fuera de casa en el resultado de las competiciones deportivas (Sampedro \& Prieto, 2012). Según Jamieson (2010), esta ventaja se sitúa en torno al 60\% en los deportes de equipo, aunque este porcentaje varía entre los distintos deportes pues no todos presentan las mismas características. Además, este mismo autor señala que la ventaja de jugar en casa es mayor en fútbol que en el resto de deportes de equipo analizados (Eugster, Gertheiss \& Kaiser, 2010; Krumer, 2017; Pic, 2018).

En este sentido, Pollard \& Armatas (2017) realizan un trabajo en el que examinan las fases finales para la clasificación de los Campeonatos del Mundo de Fútbol de los años 2006, 2010 y 2014, y demuestran que HA alcanza el 61.8\%. En esta línea, recientes estudios analizan las fases regulares de las ligas de fútbol profesional y señalan alrededor del $61 \%$ para la LaLiga (Pollard \& Gómez, 2014; Saavedra, Gutiérrez, Fernández \& Sa, 2015), el 62.29\% para la Premier League (Pollard \& Gómez, 2015), y en torno al 58\% en la Francia Ligue 1, Bundesliga y Serie A (Leite, 2017).

Fecha recepción: 23-03-18. Fecha de aceptación: 26-09-18 Francisco Daniel Martínez Martínez

fdaniel.martinez@um.es
Las principales causas que pueden explicar esta ventaja hacen referencia a la interacción de siete factores: efectos del público, territorialidad, familiaridad con el entorno de juego, parcialidad del árbitro, efecto de los viajes, factores psicológicos, y tácticos (González, ValdiviaMoral, Cachón, Zurita \& Romero, 2017; Pollard, 2008). Entre ellos, uno de los más aceptados por los aficionados, jugadores, entrenadores, árbitros y medios de comunicación es el apoyo del público (Anderson, Wolfson, Neave \& Moss, 2012). De hecho, los equipos técnicos asumen estrategias más ambiciosas al jugar como local, tratando de que sus jugadores busquen de forma activa marcar un gol en los primeros minutos del encuentro, provocando una mayor excitación e implicación del público (Collet, 2013; Duarte et al., 2013; Staufenbiel, Lobinger \& Strauss, 2015). A su vez, al aumentar la presión de los aficionados, el árbitro suele sacar más tarjetas rojas y amarillas a los jugadores del equipo visitante(Picazo-Tadeoa, González-Gómez \& Guardiola, 2017). En cualquier caso, la magnitud de $H A$ varía en función de la calidad del oponente (García-Rubio et al., 2015; Lago-Peñas, Gómez \& Pollard, 2017).

Del mismo modo, HA también oscila en la competición de ida y vuelta, al tener una gran influencia el valor de los goles y la localización del partido, dependiendo de si es el primer o el segundo encuentro el que se disputa en casa (Pic \& Castellano, 2017). Así, estos mismos autores Pic \& Castellano (2017), confirman que jugar como local el partido de vuelta aumenta las probabilidades de superar la eliminatoria, aunque este efecto varía en las diferentes rondas. En cambio, si se considera el margen de goles, cuando el club que juega en casa el primer encuentro gana con uno o más goles de margen, sus posibilidades de pasar a la siguiente fase son mayores que si vence en las mismas circunstancias el equipo que juega a domicilio (Pic \& Castellano, 2016, 2017).

En esta línea, marcar el primer gol puede crear un momento psicológico positivo que haga la victoria más probable (Jones, 2009). Por el contrario, encajar un gol puede generar un momento negativo, sacando a la afición fuera del partido (Courneya, 1990), provocando una crisis en el equipo, disminuyendo la confianza de los jugadores, aumentando la desmoralización y reduciendo la cohesión del grupo(Bar-Eli, Tenenbaum \& Geister, 2006). 
En este sentido, algunos estudios (Lago-Peñas et al., 2016; Sampedro \& Prieto, 2012) indican que cuando el equipo local abre el marcador gana alrededor del 74\% de los partidos, mientras que cuando marca primero el que juega a domicilio, el porcentaje de victorias se sitúa en el 63\%. Además, si ese primer gol se produce cuando el partido está llegando a su fin, aumenta aún más su importancia en el resultado final del encuentro (Anderson \& Sally, 2014; Lago-Peñas et al., 2016).

Por otra parte, Sampedro \& Prieto (2012) comparan la «ventaja de jugar en casa» que se produce en LaLiga y la Liga Nacional de Fútbol Sala, estableciendo un $60.21 \%$ y un $62.67 \%$ respectivamente. Además, relacionan el efecto de marcar primero y la ventaja de jugar en casa, estableciendo una nueva métrica denominada «ventaja de jugar en casa para marcar el primer gol». Este análisis permite conocer qué ventaja otorga a los equipos la localización de un partido en la consecución del primer gol. Así, en LaLiga señalan un 56.96\%, mientras que el porcentaje en la Liga Nacional de Fútbol Sala es del 57.30\%. De igual modo, en ambas competiciones analizan la «ventaja de marcar primero», siendo en LaLiga del $86.15 \%$ para el equipo local, y del $72.49 \%$ para el visitante; y en la Liga Nacional de Fútbol Sala del 75.93\% para el conjunto de casa, y del $55.77 \%$ para el que juega a domicilio.

Por otro lado, cuanto más equilibrada es una liga más interesante resulta para sus seguidores (Rottenberg, 1956). En este sentido, otros trabajos (Gasparetto \& Barajas, 2015; Lago-Peñas et al., 2016) muestran que existe una gran igualdad entre las principales ligas de fútbol europeas (LaLiga, Premier League, Francia Ligue 1, Bundesliga y Serie A), aunque no ocurre lo mismo entre los clubes de una misma liga, donde un reducido grupo de equipos dominan la tabla, provocando un descenso de la competitividad en las temporadas más recientes (SánchezFlores et al., 2016).

Por lo tanto, marcar primero y la localización del encuentro son dos variables que han suscitado el interés de la literatura científica anterior, a mismo tiempo que han mostrado una gran influencia en el resultado final del partido(García-Rubio etal., 2015; Lago-Peñas et al., 2016; Sampedro \& Prieto, 2012). Asimismo, el fútbol es un deporte que tiene un gran impacto social, mueve grandes cantidades económicas y suscita preocupación por parte de los aficionados y medios de comunicación (Borrisser \& Solanellas, 2018). Así mismo, LaLiga, Premier League, Francia Ligue 1, Bundesliga y Serie A, están catalogadas como las mejores ligas del continente europeo, lo cual acrecienta la importancia de estas competiciones a nivel mundial (Gasparetto \& Barajas, 2015; LagoPeñas et al., 2016). Teniendo en cuenta todo lo comentado, y una vez vista la influencia que tiene el efecto de marcar primero, y de jugar en casa o fuera de ella, en el resultado final de los partidos de fútbol, el presente trabajo tiene como objetivos: i) determinar la influencia del efecto de marcar primero, y la localización del encuentro en el resultado final de un partido de fútbol; y ii) contrastar la existencia de diferencias entre las principales ligas del fútbol europeo: LaLiga, Premier League, Francia Ligue 1, Bundesliga y Serie A.

\section{Método}

\section{Participantes}

La muestra estuvo formada por todos los partidos $(N=1826)$ disputados durante la temporada 2016/2017 en LaLiga $(n=380)$, Premier League $(n=380)$, Francia Ligue $1(n=380)$, Bundesliga $(n=306)$ y Serie A $(n=380)$. Se seleccionaron estas ligas por estar consideradas como las cinco mejores del continente europeo (Lago-Peñas et al., 2016). Están formadas por 20 equipos (LaLiga, Premier League, Francia Ligue 1 y Serie A) y 18 (Bundesliga); por lo que, a lo largo de la temporada, cada club juga un total de 38 partidos, o 34 en el caso de la Bundesliga, disputando el mismo número de encuentros en casa y fuera. Los datos se extrajeron de la base de datos estadísticos de fútbol http:// www.soccerstats.com/, la cual se compone de datos estadísticos de fútbol de las ligas más importantes de Europa y Sudamérica. Además, se comprobó que se podía acceder a los datos con fines de investigación. Por lo tanto, se respetaron las normas éticas para el uso de los datos.

\section{Análisis de las variables}

La ventaja de jugar en casa se produce cuando los equipos locales ganan más del $50 \%$ de los partidos en las competiciones de ida y vuelta (Courneya \& Carron, 1992), como es el caso de las ligas analizadas en este estudio, consiguiendo obtener más puntos en casa que fuera. De la misma forma, se considera que no existe ventaja de jugar en casa cuando se gana un valor igual o inferior al $50 \%$ de los encuentros, obteniendo los mismos puntos o menos al jugar como local que como visitante. Esta ventaja es calculada como el número de puntos ganados en casa por el equipo local, expresado como un porcentaje de todos los puntos conseguidos tanto en casa como fuera (Pollard, 1986).

La ventaja de jugar en casa para marcar el primer gol se da cuando se obtiene un valor superior al $50 \%$ en esta proporción; es decir, señala que los equipos locales abren el marcador en más ocasiones que los visitantes. Por el contrario, no existe ventaja de jugar en casa para marcar el primer gol si se obtiene un valor igual o inferior al 50\%. Para calcular esta ventaja, Sampedro \& Prieto (2012) adaptan el procedimiento introducido por Pollard (1986) para el cálculo de la ventaja de jugar en casa, y lo definen como el número de partidos en que los equipos locales marcan el primer gol expresado como un porcentaje de todos los partidos jugados en los que se marcan goles. Los encuentros que finalizan con empate a cero no son considerados para este análisis.

La ventaja de marcar primero para los equipos locales y visitantes se produce cuando se obtiene un valor superior al $50 \%$ en esta proporción; es decir, indica que los equipos que abren el marcador consiguen más puntos que los que encajan el primer gol. Por el contrario, no existe ventaja de marcar primero teniendo en cuenta la condición de local o visitante, si se obtiene un valor igual o inferior al $50 \%$. Esta ventaja es calculada siguiendo el procedimiento de Pollard (1986) para la ventaja de jugar en casa, entendido como el número de puntos obtenidos por los equipos que se adelantan en el marcador en función de su condición de local o visitante, expresados como un porcentaje de todos los puntos conseguidos por estos equipos (locales o visitantes) en todos los partidos disputados.

\section{Análisis de datos y procedimiento}

El tratamiento estadístico de los datos se realizó mediante el programa SPSS 19.0y los porcentajes se calcularon con Excel versión 2010. Las características de la muestra se obtuvieron a través de los estadísticos descriptivos de media, mínimo, máximo, desviación típica, frecuencia y porcentajes. El porcentaje de ventaja de jugar en casa fue calculado con la siguiente fórmula $=($ victorias en casa $\times 3+$ empates $\times 1) /($ victorias en casa $\times 3+$ empates $\mathrm{x} 1$ + victorias fuera de casa $\mathrm{x} 3$ + empates $\mathrm{x} 1)$. La ventaja de marcar primero se calculó de la siguiente manera $=$ (victorias en casa $\times 3$ + empates $\times 1$ )/(victorias en casa $\times 3$ + empates $\times 1$ + victorias fuera de casa $\mathrm{x} 3+$ empates $\mathrm{x} 1$ ). Por otro lado, la ventaja de jugar en casa para marcar el primer gol, se calculó realizando la siguiente fórmula $=\left(\mathrm{N}^{\circ}\right.$ de veces que el equipo local marca primero $) /\left(\mathrm{N}^{\circ}\right.$ total de veces que un equipo marca primero local/visitante).

En los análisis estadísticos se utilizó un error tipo I del 95\% en el empleo de las distintas pruebas estadísticas. El cálculo de las diferencias entre grupos de la misma muestra se realizó utilizando la prueba $t$ para muestras relacionadas. Para calcular las diferencias entre grupos se utilizó la prueba ANOVA de un factor. Cuando las variables fueron estadísticamente significativas, se calculó el tamaño del efecto a través de la $d$ de cohen. En este caso, siguiendo a Cohen (1988) se pueden considerar los resultados del tamaño del efecto de la siguiente forma: $d$ $=.20$ (pequeño), $d=.50$ (moderado) y $d=.80$ (grande).

\section{Resultados}

En la Tabla 1, se muestra HA en cada liga. Para ello, se ha cuantificado el número de puntos ganados en casa expresado como un porcentaje de todos los puntos ganados como local y visitante. La Francia Ligue 1 presenta los valores más altos (62.33\%) de las cinco ligas, seguida de la Bundesliga (62.08\%), Premier League (61.08\%), LaLiga (60.13\%) y 
Serie A (59.62\%). La diferencia observada entre las cinco ligas no fue significativa $(F=.52 ; p>.05)$.

\begin{tabular}{|c|c|c|c|c|c|}
\hline Liga & Partidos $(n)$ & $\begin{array}{l}\text { Victorias } \\
\text { en casa }\end{array}$ & Empates & $\begin{array}{c}\text { Victorias } \\
\text { fuera de casa }\end{array}$ & $\begin{array}{l}\text { Ventaja de jugar } \\
\text { en casa (\%) }\end{array}$ \\
\hline LaLiga & 380 & 181 & 89 & 110 & 60.13 \\
\hline Premier League & 380 & 187 & 84 & 109 & 61.08 \\
\hline Bundesliga & 306 & 150 & 74 & 82 & 62.08 \\
\hline Serie A & 380 & 184 & 80 & 116 & 59.62 \\
\hline Francia Ligue 1 & 380 & 186 & 94 & 100 & 62.33 \\
\hline Total & 1826 & 888 & 421 & 517 & 61.04 \\
\hline
\end{tabular}

La Tabla 2 muestra la distribución del resultado final (victoria, empate o derrota) de cada liga en función de si el equipo que marca primero es el local o el visitante. A nivel general, los equipos que juegan en casa y marcan el primer gol consiguen el 75.72\% de las victorias, mientras que si abre el marcador el que juega a domicilio, el porcentaje de partidos ganados se sitúa en el $61.81 \%$. Además, la ventaja de marcar primero en casa (84.65\%) es mayor con respecto a la ventaja de marcar primero fuera (75.50\%), aunque en ambos casos el equipo que marca el primer gol gana la mayoría de sus partidos. La probabilidad de ganar el equipo local cuando marca primero fue significativamente mayor con respecto a la probabilidad de ganar cuando marca primero el equipo visitante en la LaLiga ( $t=5.44 ; p<.01 ; d=.76)$, en la Premier League $(t$ $=7.38 ; p<.01 ; d=1.22)$, en la Serie $\mathrm{A}(t=4.42 ; p<.01 ; d=.64)$, en la Bundesliga $(t=6.65 ; p<.01 ; d=1.18)$, en la Francia Ligue $1(t=5.19$; $p<.01 ; d=.89)$ y en el total de la muestra $(t=12.69 ; p<.01 ; d=.91)$. La probabilidad de empatar el equipo local cuando marca primero con respecto a la probabilidad de empatar cuando marca primero el equipo visitante no fue significativa en la LaLiga $(t=-.59 ; p>.05)$, en la Premier League $(t=-1.62 ; p>.05)$, en la Serie A $(t=-1.15 ; p>.05)$, en la Francia Ligue $1(t=-1.23 ; p>.05)$, ni en la Bundesliga $(t=-1.76 ; p>.05)$, pero sí que fue significativa en el total de la muestra $(t=-2.95 ; p<.01 ; d=$ .43). La probabilidad de perder el equipo local cuando marca primero fue significativamente menor con respecto a la probabilidad de perder cuando marca primero el equipo visitante en la LaLiga $(t=-3.63 ; p<.01$; $d=.91)$, en la Francia Ligue $1(t=-3.59 ; p<.01 ; d=1.01)$, en la Premier League $(t=-2.47 ; p<.05 ; d=.72)$, en la Serie A $(t=-2.11 ; p<.05 ; d=$ $.56)$ y en el total de la muestra $(t=-6.00 ; p<.01 ; d=.72)$. Al contrario, no hubo diferencias significativas en la Bundesliga $(t=-1.61 ; p>.05)$.

En los resultados que se analizó la ventaja de marcar primero cuando el equipo marca primero dentro y fuera de casa, se encontraron

\begin{tabular}{|c|c|c|c|c|c|}
\hline Liga & $\begin{array}{l}\text { Marca } \\
\text { primero }\end{array}$ & $\begin{array}{l}\text { Resultado } \\
\text { final }\end{array}$ & $n$ & $\%$ & $\begin{array}{c}\text { Ventaja de } \\
\text { marcar primero } \\
(\%)\end{array}$ \\
\hline \multirow{6}{*}{ LaLiga } & Equipo & Victoria & 151 & 75.12 & \multirow{3}{*}{85.91} \\
\hline & local & Empate & 35 & 17.42 & \\
\hline & $n=201$ & Derrota & 15 & 7.46 & \\
\hline & Equipo & Victoria & 95 & 62.50 & \multirow{3}{*}{58.98} \\
\hline & visitante & Empate & 27 & 17.76 & \\
\hline & $n=152$ & Derrota & 30 & 19.74 & \\
\hline \multirow{6}{*}{ Premier League } & Equipo & Victoria & 162 & 78.26 & \multirow{3}{*}{86.05} \\
\hline & local & Empate & 26 & 12.56 & \\
\hline & $n=207$ & Derrota & 19 & 9.18 & \\
\hline & Equipo & Victoria & 90 & 61.64 & \multirow{3}{*}{73.95} \\
\hline & visitante & Empate & 31 & 21.24 & \\
\hline & $n=146$ & Derrota & 25 & 17.12 & \\
\hline \multirow{6}{*}{ Bundesliga } & Equipo & Victoria & 132 & 74.16 & \multirow{4}{*}{83.79} \\
\hline & local & Empate & 28 & 15.73 & \\
\hline & $n=178$ & Derrota & 18 & 10.11 & \\
\hline & Equipo & Victoria & 64 & 58.71 & \\
\hline & visitante & Empate & 27 & 24.78 & \multirow[t]{2}{*}{78.27} \\
\hline & $n=109$ & Derrota & 18 & 16.51 & \\
\hline \multirow{6}{*}{ Serie A } & Equipo & Victoria & 156 & 72.90 & \multirow{4}{*}{80.94} \\
\hline & local & Empate & 33 & 15.42 & \\
\hline & $n=214$ & Derrota & 25 & 11.68 & \\
\hline & Equipo & Victoria & 90 & 61.64 & \\
\hline & visitante & Empate & 28 & 19.18 & \multirow[t]{2}{*}{72.68} \\
\hline & $n=146$ & Derrota & 28 & 19.18 & \\
\hline \multirow{6}{*}{ Francia Ligue 1} & Equipo & Victoria & 163 & 77.99 & \multirow{3}{*}{89.15} \\
\hline & local & Empate & 37 & 17.70 & \\
\hline & $n=209$ & Derrota & 9 & 4.31 & \\
\hline & Equipo & Victoria & 90 & 63.83 & \multirow{3}{*}{75.44} \\
\hline & visitante & Empate & 28 & 19.86 & \\
\hline & $n=141$ & Derrota & 23 & 16.31 & \\
\hline \multirow{6}{*}{ Total } & Equipo & Victoria & 764 & 75.72 & \multirow{4}{*}{85.46} \\
\hline & local & Empate & 159 & 15.76 & \\
\hline & $n=1009$ & Derrota & 86 & 8.52 & \\
\hline & Equipo & Victoria & 429 & 61.81 & \\
\hline & visitante & Empate & 141 & 20.32 & \multirow[t]{2}{*}{73.57} \\
\hline & $n=694$ & Derrota & 124 & 17.87 & \\
\hline
\end{tabular}

diferencias significativas a favor del equipo local en la LaLiga ( $t=5.30$; $p<.01 ; d=.93)$, en la Premier League ( $t=5.15 ; p<.01 ; d=.97)$, en la Bundesliga ( $t=5.13 ; p<.01 ; d=.80)$, en la Serie A $(t=7.66 ; p<.01 ; d$ $=.67)$, en la Francia Ligue $1(t=4.66 ; p<.01 ; d=1.02)$, y en el total de la muestra $(t=11.45 ; p<.01 ; d=.89)$.

En la Tabla 3 aparece la ventaja de jugar en casa para marcar el primer gol de cada una de las cinco ligas estudiadas, así como la media de los resultados obtenidos en todas ellas. Como se puede comprobar, los valores más altos corresponden a la Bundesliga (62.02\%), mientras que los más bajos los obtiene la LaLiga (56.94\%). El promedio de las cinco ligas se sitúa en el 59.35\%. Posteriormente, se realizó un ANOVAde un factor en el que se examinó si existían diferencias en $H A$ para marcar el primer gol entre las diferentes ligas que componen la muestra, los resultados no mostraron diferencias significativas ( $p>.05 ; F=.52$ ).

Tabla 3

\begin{tabular}{|c|c|c|c|}
\hline Liga & \multicolumn{2}{|c|}{ Marca primero } & $\begin{array}{l}\text { Ventaja de jugar en casa para } \\
\text { marcar el primer gol (\%) }\end{array}$ \\
\hline \multicolumn{4}{|c|}{ Equipo local (n) Equipo visitante (n) } \\
\hline LaLiga & 201 & 15 & 56.94 \\
\hline Premier League & 207 & 146 & 58.64 \\
\hline Bundesliga & 178 & $10 \mathrm{~s}$ & 62.02 \\
\hline Serie A & 214 & 146 & 59.44 \\
\hline Francia Ligue 1 & 209 & 14 & 59.71 \\
\hline Total & 1009 & 69 & 59.35 \\
\hline
\end{tabular}

\section{Discusión}

El presente trabajo determina la influencia del efecto de marcar primero, y la localización del encuentro en el resultado final de un partido de fútbol; y contrasta la existencia de diferencias entre las principales ligas del fútbol europeo: LaLiga, Premier League, Francia Ligue 1, Bundesliga y Serie A.

Teniendo en cuenta los resultados encontrados, cabe destacar que, a nivel general, $H A$ de las principales ligas del fútbol europeo se sitúa en un $61.04 \%$, por lo que se confirman los hallazgos obtenidos por Jamieson (2010), que establece esta ventaja en torno al 60\% en los deportes de equipo, y los demostrados por Pollard \& Armatas (2017), quienes indican que en el fútbol la ventaja de jugar en casa alcanza el 61.8\%. En cambio, son superiores a los obtenidos por Lago-Peñas et al. (2016), quienes establecen una ventaja media del $58.81 \%$ para estas cinco ligas en la temporada 2014/2015.

Además, si se tienen en cuenta los valores específicos de cada liga, el presente estudio sitúa esta ventaja en el 60.13\% para la LaLiga y en el $61.08 \%$ para la Premier League, siendo estos resultados muy similares al 61\% que indican otros autores para LaLiga (Pollard \& Gómez, 2014; Saavedra, Gutiérrez, Fernández \& Sa, 2015), y al 62.29\% que Pollard \& Gómez (2015) señalan para Premier League. En cambio, los hallazgos encontrados para la Francia Ligue 1 (62.33\%), Bundesliga (62.08\%) y Serie A (59.62\%), difieren en mayor medida con los de Leite (2017), quien sitúa estas competiciones alrededor del $58 \%$. No obstante, cuando se examinó si existían diferencias entre ligas no se encontraron diferencias significativas, por lo que se comprueba la igualdad mostrada por otros estudios entre las principales ligas europeas (Gasparetto \& Barajas, 2015; Lago-Peñas et al., 2016).

Por otra parte, el trabajo que aquí se presenta demuestra que los equipos que juegan en casa y marcan el primer gol consiguen el $75.72 \%$ de las victorias, mientras que si abre el marcador el que juega a domicilio, el porcentaje de partidos ganados se encuentra en el $61.81 \%$. Como consecuencia, se confirman los resultados de algunos estudios (GarcíaRubio et al., 2015; Lago-Peñas et al., 2016; Sampedro \& Prieto, 2012) que indican que cuando el equipo local abre el marcador gana alrededor del $74 \%$ de los partidos, mientras que cuando marca primero el que juega fuera de casa, el porcentaje de victorias se sitúa en el 63\%.Además, la ventaja de marcar primero en casa (85.46\%) es mayor con respecto a la ventaja de marcar primero fuera (73.57\%). Estos resultados se asemejan en gran medida a los obtenidos por Lago-Peñas et al. (2016) y Sampedro \& Prieto (2012), quienes establecen alrededor del 85.5\% y del $74.37 \%$, respectivamente. 
Por tanto, se corrobora la importancia que tiene para los equipos adquirir un margen de goles a su favor, de cara a conseguir la victoria, independientemente de que disputen el encuentro en casa o fuera de ella. Estos resultados están en consonancia con los de Pic \& Castellano (2016, 2017), quienes, tras analizar las eliminatorias de la Copa del Rey del fútbol español, señalan que cuando los clubes obtienen un margen de goles en el partido de ida, las probabilidades de que alcancen la siguiente ronda se multiplican, sin importar que el de vuelta lo disputen como locales o visitantes.

Continuando con esta tendencia, cabe destacar la similitud existente entre los hallazgos encontrados en este trabajo correspondientes a la ventaja de jugar en casa para marcar el primer gol en LaLiga (56.94\%), y los que demuestran Sampedro \& Prieto (2012) para esta misma liga (56.96\%). En cambio, en el resto de ligas la ventaja es mayor, obteniendo como promedio de todas ellas un $59.35 \%$, aunque no se han encontrado diferencias significativas entre las mismas, mostrándose de nuevo la igualdad existente en las ligas examinadas en la muestra (Gasparetto \& Barajas, 2015; Lago-Peñas et al., 2016). En este sentido, podría ser interesante seguir investigando en esta línea debido a que no se han encontrado estudios con los que comparar estos resultados, por lo que se consideran un aporte científico relevante en este ámbito.

Como líneas de propuesta futura, sería interesante analizar la evolución que ha tenido $H A$ en los últimos años, así como comparar si esta ventaja se da de la misma forma en torneos eliminatorios como la fase final de la UEFA Champions League, de la Europa League, o de torneros similares. Además, también sería interesante comparar estos análisis en otros deportes de equipo como puede ser el balonmano, hockey o el rugby, para comprobar cómo evolucionan estos marcadores en deportes distintos y con menor repercusión mediática.

En cuanto a las limitaciones, se debe tener cautela a la hora de comparar estos resultados con ligas de menor nivel o de otros continentes, así como con los campeonatos eliminatorios que se deciden con partido de ida y vuelta, ya que las características del tipo de competición pueden modificar los hallazgos que se han obtenido en esta investigación. Además, también se debe tener presente que las ventajas analizadas en este estudio pueden variar en función de la calidad del oponente.

Como conclusiones, en este trabajo se demuestra el gran equilibrio existente entre las principales ligas del fútbol europeo, así como la importancia que tiene para un equipo marcar el primer gol, ya sea en casa o a domicilio, pues le garantiza una alta probabilidad de obtener un buen resultado al final del partido. Del mismo modo, la localización del encuentro se considera una ventaja que los clubes deben aprovechar, por lo que si se combinan ambas variables el éxito está prácticamente asegurado. En este sentido, los equipos técnicos deben utilizar esta información para trabajar en los entrenamientos estrategias que les permitan salir al terreno de juego con la total predisposición de abrir el marcador, así como dotar a sus jugadores de herramientas y mecanismos con las que abordar con éxito las situaciones del partido que se dan cuando reciben un gol en contra.

\section{Referencias}

Anderson, C. \& Sally, D. (2014). The Numbers Game: Why Everithing You Know abou Football Is Wrong. New York: Penguin Books.

Anderson, M., Wolfson, S., Neave, N. \& Moss, M. (2012). Perspectives on the home advantage: A comparison of football players, fans and referees. Psychology of Sport and Exercise, 13(3), 311-316.

Bar-Eli, M., Tenenbaum, G \& Geister, S. (2006). Consequences of players' dismissal in professional soccer: A crisis-related analysis of group-size effects. Journal of Sports Sciences, 24(10), 1083-1094.

Borrisser, J. \& Solanellas, F. (2018). Estudio comparativo del patrocinio de equipos profesionales en España. El caso del futbol, baloncesto, balonmano y hockey. Retos, 34, 205-211.

Casal, C. A., Losada, J. L., Maneiro, R. \& Ardá, T. (2017). Influencia táctica del resultado parcial en los saques de esquina en fútbol. Revista Internacional de Medicina y Ciencias de la Actividad Física y el Deporte, 17(68), 715-728.

Castellano, J. (2009). Conocer el pasado del fútbol para cambiar su futuro. Acción motriz, 2, 1-19.

Cohen, J. (1988). Statistical Power Analysis for the Behavioral Sciences. New York: Academic Press.

Collet, C. (2013). The possession game? A comparative analysis of ball retention and team success in European and international football, 2007-2010. Journal of Sports Sciences, 31(2), 123-136.

Courneya, K. S. (1990). Importance of game location and scoring first in college baseball. Perceptual and Motor Skills, 71(2), 624-626.

Courneya, K. S. \& Carron, A. V. (1992). The home advantage in sport competitions: A literature review. Journal of Sport \& Exercise Psychology, 14(1), 13-27.

Duarte, R., Araújo, D., Folgado, H., Esteves, P., Marques, P. \& Davids, K. (2013). Capturing complex, non-linear team behaviours during competitive football performance. Journal of Systems Science and Complexity, 26(1), 62-72.

Eugster, M. J., Gertheiss, J. \& Kaiser, S. (2010). Having the second leg at

home - advantage in the UEFA champions league knockout phase? Journal of

Quantitative Analysis in Sports, 7(1), 1-9.

García-Rubio, J., Gómez, M. Á.,Lago-Peñas, C. \& Ibáñez, J. S. (2015). Effect of match venue, scoring first and quality of opposition on match outcome in the UEFA Champions League. International Journal of Performance Analysis in Sport, 15(2), 527-539.

Gasparetto, T. \& Barajas, A. (2015). Análisis del balance competitivo por la diferencia de puntos acumulada (DPA). Proceedings of XXV Jornadas Hispanolusas. Ourense: España.

González, G, Valdivia-Moral, P., Cachón, J., Zurita, F. \& Romero-Ramos, O. (2017). Influencia del control del estrés en el rendimiento deportivo: la autoconfianza, la ansiedad y la concentración en deportistas. Retos, 32, 3-6.

Jamieson, J. P. (2010). The home field advantage in athletics: A meta analysis. Journal of Applied Social Psychology, 40(7), 1819-1848.

Jones, B. M. (2009). Scoring first and home advantage in the NHL. International Journal of Performance Analysis in Sport, 9(3), 320-331.

Krumer, A. (2017). On winning probabilities, weight categories, and home advantage in professional judo. Journal of Sports Economics, 18(1), 77-96.

Lago-Peñas, C., Gómez-Ruano, M., Megías-Navarro, D. \& Pollard, R. (2016). Home advantage in football: Examining the effect of scoring first on match outcome in the five major European leagues. International Journal of Performance Analysis in Sport, 16(2), 411-421.

Lago-Peñas, C., Gomez, M. Á. \& Pollard, R. (2017). Home advantage in elite soccer matches. A transient effect? International Journal of Performance Analysis in Sport, 17(1-2), 86-95.

Leite, W. S. (2017). Home advantage: Comparison between the major European football leagues. Athens Journal of Sports, 4(1), 65-74.

López-García, S., Maneiro, R., Ardá, A., Rial, A., Losada, J. L. \& Casal-Sanjurjo, C.A. (2018). Tiros libres indirectos en fútbol de alto nivel. Identificación de variables explicativas. Revista Internacional de Medicina y Ciencias de la Actividad Física y el Deporte,18(70), 247-268.

Page, L. \& Page, K. (2007). The second leg home advantage: Evidence from European football cup competitions. Journal of Sports Sciences, 25(14), 1547-1556.

Pic, M. (2018). Temporal consistencies in two champion teams of European football? Retos, 34(2), 94-99.

Pic, M., \& Castellano, J. (2016). Efecto de la localización del partido en eliminatorias de ida y vuelta de la UEFA Champions League. Revista Internacional de Ciencias del Deporte, 44(12), 149-163.

Pic, M. \& Castellano, J. (2017). Influence of match location in the Spanish Copa de Rey. Retos, 31, 202-206.

Picazo-Tadeo, A. J., González-Gómez, F. \& Guardiola, J. (2017). Does the crowd matter in refereeing decisions? Evidence from Spanish soccer. International Journal of Sport and Exercise Psychology, 15(5), 447-459.

Pollard, R. (1986). Home advantage in soccer: A retrospective analysis. Journal of Sports Sciences, 4(3), 237-248.

Pollard, R. (2008). Home advantage in football: A current review of an unsolved puzzle. The open sports sciences journal, 1(1), 12-14.

Pollard, R. \& Armatas, V. (2017). Factors affecting home advantage in football World Cup qualification. International Journal of Performance Analysis in Sport, 17(1-2), 121-135.

Pollard, R. \& Gómez, M. A. (2014). Comparison of home advantage in men's and women's football leagues in Europe. European journal of sport science, 14(sup1), S77S83.

Pollard, R. \& Gómez, M. Á. (2015). Comparison of home advantage in college and professional team sports in the United States. Collegium antropologicum, 39(3), 583-589.

Rottenberg, S. (1956). The baseball players' labor market. Journal of political economy, 64(3), 242-258.

Saavedra, M., Gutiérrez, O., Fernández, J. J. \& Sa, P. (2015). Measuring home advantage in Spanish football (1928-2011). Revista Internacional de Medicina y Ciencias de la Actividad Física y el Deporte, 15(57), 181-194.

Sampedro Molinuevo, J. \& Prieto Bermejo, J. (2012). El efecto de marcar primero y la ventaja de jugar en casa en la liga de fútbol y en la liga de fútbol sala de España. Revista de psicología del deporte, 21(2), 301-308.

Sánchez-Flores, J., Martín-González, J. M., García-Manso, J. M., de Saa, Y., Arriaza-Ardiles, E. J. \& da Silva-Griglotetto, M. E. (2016). Análisis de los goles conseguidos en 13 temporadas (2000/01-2012/13) correspondientes a la Primera División de la Liga Española de Fútbol Profesional. Revista Andaluza de Medicina del Deporte, 9(2), 55-61.

Soccer Stats (2017). Statistics and results on national and international football competitions. Recuperado el 5 de Enero de 2018 de http://www.soccerstats.com/

Staufenbiel, K., Lobinger, B. \& Strauss, B. (2015). Home advantage in soccer-A matter of expectations, goal setting and tactical decisions of coaches? Journal of Sports Sciences, 33(18), 1932-1941. 